\title{
Concentrações sangüíneas de lactato em eqüinos durante a prova de fundo do concurso completo de equitação
}

\author{
Blood lactate concentrations of horses competing in the resistance phase of 3-day \\ combined training event
}

\author{
Lina Maria Wehrle Gomide ${ }^{1}$ Carla Braga Martinss ${ }^{1}$ Cesar Andrey Galindo Orozco $^{1}$ \\ Rita de Cássia de Lima Sampaio ${ }^{1}$ Taisa Belli $^{2}$ Vilmar Baldissera ${ }^{2}$ \\ José Corrêa de Lacerda Neto ${ }^{3^{*}}$
}

\section{RESUMO}

Colheu-se sangue de 13 eqüinos competindo na prova de Fundo do Concurso Completo de Equitação, categoria internacional três estrelas, e dosou-se lactato sangüíneo na manhã antes da prova, antes e imediatamente depois das fases $B$ e $D$ e 10 minutos após a fase D. Foi observado aumento significativo nas concentrações de lactato apenas após o término da fase $D$, demonstrando que os animais foram submetidos a um grande esforço físico, com desenvolvimento de acidose metabólica decorrente do acúmulo de lactato. Houve diminuição significativa dos valores dez minutos após o término do cross-country, exceto para o eqüino de número 12, único animal a completar o percurso dentro do tempo estipulado, o qual apresentou sinais clínicos de exaustão.

Palavras-chave: eqüino, lactato, cross-country, steeplechase.

\section{ABSTRACT}

Blood samples were collected from thirteen horses competing in the resistance phase of an advanced 3-day-event. Blood lactate was determined in the morning before competition, before and immediately after phases $B$ and $D$, and 10 minutes after phase D. Significant increase in blood lactate was observed only after phase $D$, showing that animals were submitted to a great physical effort with development of metabolic acidosis due to lactate accumulation. There was significant decrease in blood lactate levels 10 minutes after cross country except horse 12, the only animal to finish phase $D$ inside the given time, but showed clinical signs of exhaustion.

Key words: equine, lactate, cross-country, steeplechase.

\section{INTRODUÇÃO}

O Concurso Completo de Equitação (CCE), regido pelas regras da Federação Eqüestre Internacional (FEI), é uma modalidade eqüestre que exige muito dos animais, tanto na parte de adestramento quanto no condicionamento físico (WILLIAMSON et al., 1996; WHITE et al., 1995a). O Concurso Completo Internacional, classificado de uma a quatro estrelas (CCI* - CCI****) de acordo com o nível de exigência, é o mais sofisticado dos concursos, sendo cada prova realizada obrigatoriamente em um dia, totalizando três dias de competição. No primeiro dia, ocorre a prova de adestramento, no segundo, a prova de fundo e, no terceiro, a prova de salto. A prova de fundo possui quatro fases: fase de resistência ou caminhos e trilhas (fases A e C), steeplechase (fase B) e cross-country (fase D), com paradas obrigatórias de um minuto entre as fases $A$ e $B$, de 5 a 10 minutos durante a fase $C$ e de 10 minutos entre as fases C e D para inspeção veterinária.

A produção e a utilização apropriadas de energia são essenciais para o eqüino atleta e possuem uma função crítica para o ótimo desempenho (EATON, 1994; HARRIS \& HARRIS, 1998). A glicose é uma importante fonte de energia para a atividade muscular.

\footnotetext{
${ }^{1}$ Faculdade de Ciências Agrárias e Veterinária (FCAV), Universidade Estadual Paulista (UNESP), Jaboticabal, SP, Brasil. ${ }^{2}$ Departamento de Ciências Fisiológicas, Universidade Federal de São Carlos (UFSCAR), São Carlos, SP, Brasil.

3*Departamento de Clínica e Cirurgia Veterinária, FCAV/UNESP, Jaboticabal, SP, Brasil. Via de Acesso Prof. Paulo Donato Castellane, s/n, 14884-900. Email: jlacerda@fcav.unesp.br. Autor para correspondência.
} 
Com o aumento da intensidade do exercício, grande parte da energia é gerada através da glicólise anaeróbia, com conseqüente produção de ácido láctico. Quanto maior a intensidade do exercício, maior a quantidade de lactato e $\mathrm{H}^{+}$produzidos (EATON, 1994). Durante a prova de fundo, os animais realizam exercícios extenuantes, sendo considerada um dos testes mais desafiadores do condicionamento e habilidade dentre as modalidades eqüestres (WILLIAMSON et al., 1996; MARLIN et al., 1995). MUNOZ et al. (1999) relatam que a energia muscular durante o cross-country é originada tanto pelo processo oxidativo quanto pela glicólise anaeróbia, com conseqüente produção de lactato. WILLIAMSON et al. (1996), ANDREWS et al. (1995) e MARLIN et al. (1995) demonstraram que eqüinos, após a prova de fundo, apresentaram várias alterações bioquímicas, relacionadas principalmente à perda de líquido e eletrólitos através do suor, além de acidose metabólica relacionada ao acúmulo de lactato no sangue. Dessa forma, é indispensável o adequado condicionamento físico destes animais.

O acúmulo de lactato no músculo, e conseqüente acidose intracelular, pode prejudicar a glicólise, a capacidade respiratória da mitocôndria (COUROUCÉ, 1998) e estar relacionado a uma falha em manter a homeostase ADP/ATP no sítio de ligação miosina-actina. $\mathrm{O}$ aumento de ADP local pode causar perda de performance por fadiga muscular, sendo o principal impedimento para a continuidade do trabalho (HARRIS \& HARRIS, 1998). Um dos efeitos do treinamento é aumentar a intensidade do exercício na qual o lactato começa a se acumular (limiar anaeróbico), além de melhorar a capacidade cardio-respiratória do animal (EATON et al., 1999; COUROUCÉ, 1998).

A concentração de lactato sangüíneo ou sérico vem sendo utilizada com tanta freqüência quanto os parâmetros clínicos e fornece informações adicionais sobre o condicionamento atual do atleta (LINDNER, 2000). Segundo MARLIN \& NANKERVIS (2002), testes de performance a campo são mais específicos e realistas, principalmente se forem similares às condições de competição. A concentração de lactato sangüíneo é uma variável de fácil aferição, mesmo em condições de campo (COUROUCÉ, 1998) e está relacionada à intensidade do exercício, possibilitando avaliar o sistema de produção energética mais utilizado (DESMECHT et al., 1996). A concentração de lactato é a variável que apresenta melhor correlação com a performance competitiva do animal (LINDNER, 2000).

Vários estudos foram realizados em outros países, principalmente com o objetivo de obter informações para que a realização dos Jogos Olímpicos, em condições ambientais estressantes, fosse mais segura para os animais. Entretanto, a literatura nacional é escassa e, segundo nosso conhecimento, este é o primeiro trabalho a avaliar eqüinos de CCI durante a competição. Assim, este estudo teve como objetivo obter informações sobre o condicionamento de eqüinos atletas de elite e avaliar a intensidade de esforço ao qual os animais são submetidos durante a prova de Fundo de um $\mathrm{CCI}^{* * *}$ no nosso país.

\section{MATERIAL E MÉTODOS}

Foram utilizados 13 eqüinos de raça, sexo e idade variados, que estavam competindo durante uma prova de CCI***, seletiva para os Jogos Olímpicos de Athenas 2004, durante o segundo dia de competição ou prova de fundo. A prova foi realizada na cidade de São Carlos, estado de São Paulo, Brasil. As distâncias e velocidades exigidas na competição B e D estão expressas na tabela 1. A temperatura ambiental média e a umidade relativa do ar média foram, respectivamente, 23,5ㄷ $\mathrm{C} 69 \%$.

As amostras de sangue foram tomadas pela manhã com os animais em repouso (basal), imediatamente antes e ao término das fases B e D e 10 minutos após o término da fase D. Todas as colheitas, exceto a basal, coincidiram com a inspeção veterinária dos animais. Para a colheita, foram utilizadas seringas de $3 \mathrm{~mL}$ com agulhas $25 x 7$ e o sangue diluído em fluoreto de sódio a $1 \%$ na proporção de 1:2. As amostras permaneciam no gelo até a determinação das concentrações sangüíneas de lactato ${ }^{a}$, o que ocorreu em até 10 minutos.

Análise estatística

Os valores médios obtidos foram comparados pelo Teste t-Student, com nível de significância $\mathrm{P} \leq 0,05$.

Tabela 1 - Característica, distância percorrida e velocidade exigida em cada fase da prova de fundo do concurso completo de equitação. SP: São Carlos, 2004.

\begin{tabular}{llccc}
\hline Fase & Característica & Distância $(\mathrm{m})$ & Número de obstáculos & Velocidade (m/min) \\
\hline A & Caminho e trilhas & 3960 & - & 220 \\
B & Steeplechase & 2415 & 8 & 690 \\
C & Caminhos e trilhas & 560 & - & 160 \\
D & Gross- country & 5415 & 40 & 570 \\
\hline
\end{tabular}




\section{RESULTADOS E DISCUSSÃO}

Dos 13 animais que iniciaram a prova de fundo, somente onze completaram todas as fases. Um dos conjuntos foi eliminado na inspeção veterinária antes da fase D por manifestar claudicação e outro conjunto apresentou uma queda durante o percurso do cross-country. Dentre os conjuntos que completaram a prova de fundo, o animal de número 12 foi o único a concluir o percurso da fase D no tempo determinado, enquanto os demais ultrapassaram o limite de tempo. Com isso, este eqüino apresentou respostas fisiológicas nitidamente diferentes dos outros animais após a fase $\mathrm{D}$, formando-se então dois grupos: um grupo chamado GI, referente aos 12 animais que apresentaram respostas fisiológicas semelhantes, obtendo-se a média dos valores obtidos, e outro referente ao animal número 12. Os resultados das concentrações sangüíneas de lactato estão expressos na tabela 2 e na figura 1 . Houve aumento, embora não significativo, nos valores de lactato sangüíneo ao final da fase B (steeplechase). Após a fase $\mathrm{C}$ (caminhos e trilhas) observou-se uma diminuição significativa nas concentrações de lactato. Ao término da fase D (cross-country), houve um aumento significativo, com valores superiores aos obtidos após a fase B. Nos animais do grupo G-I, houve uma diminuição significativa do lactato sangüíneo dez minutos após o final desta fase. Ao término da prova, o animal de $\mathrm{n}^{\mathrm{o}} 12$ apresentou elevação persistente de temperatura retal, aumento das freqüências cardíaca e respiratória, dilatação do ânus e rigidez muscular, mostrando sinais evidentes de exaustão. Estes dados foram acompanhados pela incapacidade de reduzir a

Tabela 2 - Média e erro padrão da concentração de lactato sangüíneo (mmol/L) nos diferentes tempos e fases estudadas do grupo GI e concentração de lactato sangüíneo do animal 12 no Concurso Completo de Equitação. SP: São Carlos, 2004.

\begin{tabular}{lcc}
\hline Momento & Grupo I (média \pm EPM) & Animal 12 \\
\hline Basal & $1,50 \pm 0,05^{\mathrm{d}}$ & 1,06 \\
Pré- B & $1,40 \pm 0,10^{\text {de }}$ & 1,20 \\
Pós- B & $6,95 \pm 1,54^{\text {be }}$ & 7,83 \\
Pré- D & $2,36 \pm 0,18^{\mathrm{c}}$ & 1,83 \\
Pós- D & $11,57 \pm 0,84^{\mathrm{a}}$ & 21,51 \\
$10^{\prime}$ pós- D & $8,74 \pm 0,87^{\mathrm{b}}$ & 24,96 \\
\hline
\end{tabular}

Basal = antes da prova de fundo; Pré-B = antes do steeplechase; Pós-B = após o steeplechase; Pré-D = antes do cross-country; Pós-D = após o cross-country; 10' pós- $\mathrm{D}=10$ minutos após a prova de fundo.

Médias seguidas de letras diferentes na mesma coluna diferem pelo teste t-Student $(\mathrm{p} \leq 0,05)$. concentração sangüínea de lactato 10 minutos após o término do cross-country.

O fato das concentrações sangüíneas de lactato apresentarem elevações significativas durante o cross-country indica que o esforço ao qual os animais foram submetidos durante esta fase foi mais intenso. Apesar de os animais do grupo GI apresentarem diminuição nos valores de lactato dez minutos após o término do exercício e do eqüino número 12 continuar em ascensão, não é possível inferir que os animais do grupo GI possuíam melhor condicionamento físico, já que o eqüino 12 foi submetido a maior esforço, sendo o único a terminar o cross-country dentro do tempo máximo permitido.

Autores que avaliaram as freqüências cardíacas durante e as concentrações de lactato após o cross-country (MUNOZ et al., 1999; MARLIN et al., 1995; WHITE et al., 1995a; WHITE et al.,1995b) e autores que avaliaram somente as concentrações de lactato após a fase D (ANDREWS et al., 1995; WILLIAMSON et al., 1996) demonstraram que ocorre uma elevada taxa de metabolismo anaeróbico para suprir as demandas energéticas durante o exercício, com conseqüente acúmulo de lactato e acidose metabólica. AGUILERA-TEJERO et al. (1998) demonstraram que eqüinos de salto, apesar de realizarem provas com intensidade e duração baixas, apresentam acúmulo de lactato e uma pequena perda de líquido após o exercício, indicando que, para transpor os obstáculos, os animais são submetidos a um grande esforço. Estes autores observaram um valor máximo de lactato de 5,3mmol L-1 . No entanto, se considerar-se o grande aumento na distância do percurso, na velocidade exigida para a realização da fase D e a dificuldade dos obstáculos, facilmente atingir-se-á as concentrações encontradas neste estudo. Os resultados do grupo G-I são inferiores aos citados por outros autores após a fase D (WILLIAMSON et al., 1996; ANDREWS et al., 1995; MARLIN et al., 1995; WHITE et al., 1995a; WHITE et al., 1995b), que obtiveram valores plasmáticos médios de 18,8, 18,4, 22,4, 21,3 e 19,1 $\mathrm{mmol} \mathrm{L}^{-1}$ respectivamente. Essa diferença pode estar relacionada à intensidade do exercício, ao condicionamento dos animais e também ao fato de que os dados da literatura foram obtidos no plasma e não no sangue total, o que fornece resultados numericamente maiores.

Após dez minutos do término do crosscountry, ocorreu redução na temperatura retal dos animais. Estes dados contrariam os achados de WHITE et al. (1995a), que relatou aumento da temperatura retal em todos os animais estudados até dez minutos após o exercício. Provavelmente esta queda da temperatura 


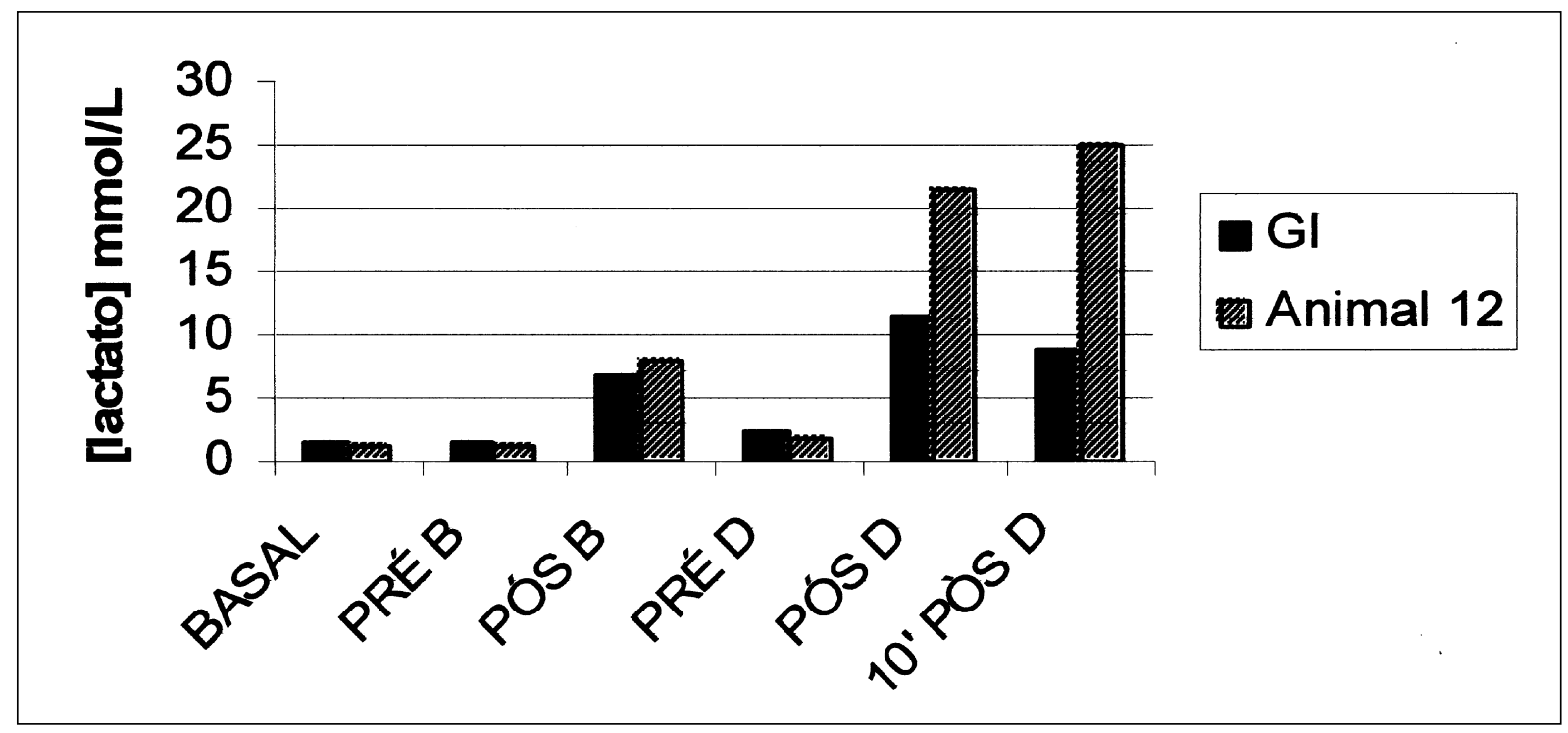

Figura 1 - Média da concentração de lactato sangüíneo (mmol L-1) nos diferentes tempos e fases estudadas, do grupo GI e do animal 12, no Concurso Completo de Equitação. SP: São Carlos, 2004.

Basal: antes da prova de fundo; Pré- B: antes do steeplechase; Pós- B: após o steeplechase; Pré- D: antes do cross - country; Pós- D: após o croos country; 10’ pós- D: 10 minutos após a prova de fundo.

ocorreu devido ao resfriamento realizado com água fria em todos os animais após a fase $\mathrm{D}$, indicando que este procedimento é altamente benéfico para a dissipação do calor produzido durante o exercício.

A redução das concentrações de lactato entre as fases B e D mostra que a fase C é essencial para a recuperação cardiovascular e para a dissipação do lactato acumulado durante a fase B (WILIAMSON et al., 1996; WHITE et al., 1995b), permitindo que o animal comece a fase $\mathrm{D}$, que é a mais extenuante, em melhores condições metabólicas.

Os dados obtidos mostram que a determinação das concentrações de lactato pode ser utilizada para distinguir diferentes tipos de exercício e melhor compreender a fisiologia dos eqüinos durante o exercício, conforme citado por DESMECHT et al. (1996). Apesar da variedade de raças e seus cruzamentos utilizados em provas de CCE, PRINCE et al. (2002) demonstraram que, mesmo com as diferenças metabólicas entre as raças Puro Sangue Inglês e Puro Sangue Árabe, quando submetidos ao mesmo teste, os animais não apresentaram diferenças significativas nas concentrações de lactato plasmático após o exercício.

\section{CONCLUSÃO}

Pode-se concluir que a determinação das concentrações de lactato ao final da prova e da capacidade do organismo em removê-lo permitiu inferir sobre o esforço físico ao qual os animais foram submetidos e no preparo destes animais para realizar o tipo de exercício imposto. Os animais do grupo GI realizaram grande esforço físico, mas este encontravase dentro dos limites de cada animal. Já as respostas fisiológicas do eqüino número 12, tanto na incapacidade de metabolizar o lactato quanto no desenvolvimento de quadro clínico de exaustão, indicam que o esforço físico imposto a este animal foi excessivo.

\section{AGRADECIMENTOS}

À Confederação Brasileira de Hipismo (CBH) e à Federação Paulista de Hipismo (FPH), pela oportunidade e aos proprietários por disponibilizarem seus animais para este estudo.

À Fundação de Amparo à Pesquisa do Estado de São Paulo (FAPESP), pela aquisição do lactímetro através do Processo 03/13445-9.

\section{FONTESDEAQUISIÇÃO} Incorporated)

a - Lactímetro YSL 1500 Sport (Yellow Springs

\section{REFERÊNCIAS}

AGUILERA-TEJERO, E. et al. Acid-base balance after exercise in show jumpers. In: CONFERENCE ON EQUINE SPORTS MEDICINE AND SCIENCE, 1998, Cordoba, Espanha. Anais... The Netherlands: Wageningen Pers, 1998. 272p. p.43-45.

ANDREWS, F.M. et al. Haematological and bioquemical changes in horses competing in a 3 Star horse trial and 3-dayevent. Equine Veterinary Journal, Supl.20, p.57-63, 1995.

Ciência Rural, v.36, n.2, mar-abr, 2006. 
COUROUCÉ, A. Endurance and sprint training. In: CONFERENCE ON EQUINE SPORTS MEDICINE AND SCIENCE, 1998, Cordoba, Espanha. Anais... The Netherlands: Wageningen Pers, 1998. 272p. p.190-202.

DESMECHT, D. et al. Relationship of plasma lactate production to cortisol release following completion of different types of sporting events in horses. Veterinary Research Communications, v.20, n.4, p.371-379, 1996.

EATON, M.D. Energetics and performance. In: HODGSON, D.R.; ROSE, R.J. The athletic horse: principles and practice of equine sports medicine. Philadelphia: Saunders. 1994. p.4962.

EATON, M.D. et al. Effects of low- and moderate-intensity training on metabolic responses to exercise in Thoroughbreds. Equine Veterinary Journal, Supl.30, p.521-527, 1999.

HARRIS, P.A.; HARRIS, R.C. Nutritional ergogenic aids in the horse - uses and abuses. In: CONFERENCE ON EQUINE SPORTS MEDICINE AND SCIENCE, 1998, Cordoba, Espanha. Anais... The Netherlands: Wageningen Pers, 1998. 272p. p.203-218.

LINDNER, A. Use of blood biochemistry for positive performance diagnosis of sports horses in practice. Revue Médecine Vétérinaire, v.151, n.7, p.611-618, 2000

MARLIN, D.J. et al. Physiological, metabolic and biochemical responses of horses competing in the speed and endurance phase of a CCI ${ }^{* * * * *}$ 3-day-event. Equine Veterinary Journal, Supl.20, p.37-46, 1995.

MARLIN, D.; NANKERVIS, K. Indicators of performance. In: MARLIN, D.; NANKERVIS, K. Equine exercise physiology. Great Britain: Blackwell, 2002. p.245-260.

MUNOZ, A. et al. Cardiovascular and metabolic adaptations in horses [Equus caballus] competing in cross-country events. Journal of Veterinary Medical Science, v.61, n.1, p.1320, 1999.

PRINCE, A. et al. Comparison of the metabolic responses of trained Arabians and Thoroughbreds during high- and lowintensity exercise. Equine Veterinary Journal, Supl.34, p.95-99, 2002.

WILLIAMSON, L.H. et al. Biochemical changes in three-dayevent horses at the beginning, middle and end of Phase $C$ and after Phase D. Equine Veterinary Journal, Supl.22, p.9298, 1996.

WHITE, S.L. et al. Heart rate response and plasma lactate concentrations of horses competing in the cross-country phase of combined training events. Equine Veterinary Journal, Supl.20, p.47-51, 1995a.

WHITE, S.L. et al. Heart rate response and plasma lactate concentrations of horses competing in the speed and endurance phase of 3-day combined training events. Equine Veterinary Journal, Supl.20, p.52-56, 1995b. 